\title{
COMUNICAÇÃO
}

\section{CONTROLE DO CARUNCHO Callosobruchus maculatus (Fabr., 1775) (Coleóptera: Bruchidae) UTILIZANDO EXTRATOS DE Piper nigrum L. (Piperaceae) PELO MÉTODO DE VAPOR}

\author{
Control of cowpea weevil Callosobruchus maculatus (Fabr., 1775)(Coleóptera: Bruchidae) \\ using extracts of Piper nigrum L. (Piperaceae) by the steam method
}

\author{
Silvana Alves de Almeida ${ }^{1}$, Francisco de Assis Cardoso de Almeida ${ }^{3}$, Nilene Rodrigues dos Santos ${ }^{1}$, \\ Sheila Soraia Araújo Medeiros ${ }^{1}$, Harley da Silva Alves $^{2}$
}

\begin{abstract}
RESUMO
Considerando os prejuízos causados pelo inseto-praga Callosobruchus maculatus (Fabr., 1775) ao feijão Vigna unguiculata Walp. armazenado, com o presente trabalho objetivou-se avaliar a eficácia de extratos de Piper nigrum L. na mortalidade de adultos daquela espécie. O delineamento estatístico utilizado foi inteiramente ao acaso com quatro repetições e arranjo fatorial 3 x 5 , constituído por três concentrações do extrato e cinco períodos de exposições dos extratos (5, 10, 15, 20 e 25 minutos). Utilizaram-se frutos secos triturados de $P$. nigrum para extração em percolador com solvente álcool etílico (70, 50 e $30 \%$ ). Os extratos foram aplicados na forma de vapor, por meio de um compressor adaptado, para dentro de recipiente contendo 100 insetos. Os resultados permitiram concluir que a mortalidade dos insetos aumenta com o aumento do período de exposição aos extratos, e que todas as concentrações se mostraram eficientes, embora, em termos de valores absolutos, o extrato com $70 \%$ de álcool etílico foi o mais eficaz.
\end{abstract}

Termos para indexação: Inseto-praga, Vigna unguiculata, armazenamento, Piper nigrum.

\begin{abstract}
Considering the damage caused by the insect pest Callosobruchus maculatus (Fabr., 1775) to stored Vigna unguiculata Walp beans, this work aimed to evaluate the effectiveness of extracts of Piper nigrum L. in the control of adults of the referred species. The statistical outline used was made at random with four repetitions and factorial arrangement $3 \times 5$, formed by three concentrations of the extract and five periods of exposure to the extracts $(5,10,15,20$ and 25 minutes). P. nigrum grounded dry fruits were used for extraction, which was done with ethyl alcohol (70,50 and 30\%), using a percolator. The extracts were applied as steam, using an adapted compressor into a container having 100 insects. By the results it was possible to conclude that the mortality of the insects becomes higher as the period of exposure to the extracts is increased, and that all the concentrations were shown to be effective, although, in terms of absolute values, the extract with $70 \%$ of ethyl alcohol was the most effective one.
\end{abstract}

Index terms: Insect pest, Vigna unguiculata, storage, Piper nigrum.

(Recebido para publicação em 28 de abril de 2003 e aprovado em 23 de abril de 2004)

O Callosobruchus maculatus (Fabr., 1775), devido ao seu potencial depreciativo e ocorrência mundial, é considerado a principal praga do feijão Vigna armazenado, reduzindo o peso e a qualidade dos grãos, bem como o poder germinativo e qualidade das sementes (DONGRE et al., 1996). Este caruncho pode ocasionar perda de peso da ordem de $60 \%$ em sementes armazenadas (TANZUBIL, 1991), chegando a atingir, em seis meses de armazenamento, $90 \%$ de perdas em termos de sementes perfuradas (SECK et al., 1991).

$\mathrm{O}$ ataque de insetos em sementes armazenadas constitui um problema que se agrava cada vez mais devido, principalmente, ao desconhecimento dos produtores quanto à utilização e manuseio de substâncias químicas (FARONI et al., 1995). O controle químico na forma de fumigação tem sido uma prática corriqueira na desinfestação de grãos armazenados, mas devido à inobservância das práticas corretas, à aplicação de subdosagens e o desrespeito ao tempo mínimo de contato dos inseticidas, vários insetos-praga que infestam produtos armazenados vêm desenvolvendo resistência genética aos princípios ativos dos produtos utilizados.

\footnotetext{
${ }^{1}$ Mestre em Engenharia Agrícola - Universidade Federal de Campina Grande/UFCG - Rua Aprígio Veloso, 882 - Bodocongó - 58.109-900 - Campina Grande, PB.

${ }^{2}$ Mestre em Química de Produtos Naturais e Sintéticos Bioativos - Cidade Universitária - Campus I - 58.059-900.

${ }^{3}$ Professor do Departamento de Engenharia Agrícola -Universidade Federal de Campina Grande/UFCG - Rua Aprígio Veloso, 882 - Bodocongó 58.109-900 - Campina Grande, PB.
} 
Esses fatos conduzem à necessidade de se estabelecer medidas de controle de pragas, por meio de métodos alternativos, sem desencadear os problemas causados pelos inseticidas sintéticos químicos (FARONI et al., 1995).

Com a utilização de métodos alternativos procurase encontrar técnicas que permitam manejar a resistência desenvolvida pelas pragas aos praguicidas organosintéticos, assim como estratégias que proporcionem uma opção de controle, que evitem a eliminação dos inimigos naturais, a contaminação da água, do ar e do solo, a intoxicação dos produtores e, especialmente, a acumulação de substâncias tóxicas nos produtos agrícolas alimentícios (CUNHA, 2002). Uma das técnicas em evidência é o uso de plantas com propriedades inseticidas na forma de pós, extratos e óleos.

De acordo com Quarles (1992), extratos botânicos apresentam algumas vantagens sobre pesticidas sintéticos, tais como: oferecer novos compostos que as pragas ainda não podem inativar; menos concentrados e, portanto, potencialmente menos tóxicos do que compostos puros; biodegradação rápida e múltiplos modos de ação, tornando possível um amplo espectro de uso enquanto retêm uma ação seletiva dentro de cada classe de praga; ademais, são derivados de recursos renováveis, diferentemente dos materiais sintéticos.

Considerada uma das espécies mais antigas, o Piper nigrum L., Piperaceae, trepadora originada da Índia, é muito cultivada nas regiões tropicais. Seus frutos, conhecidos como pimenta do reino, são miúdos e redondos de coloração roxa quase negra. Segundo Costa (1994), entre seus componentes reconhecidos encontram-se alcalóides (5,5 a $9 \%$ ) e a piperina, uma base solúvel em álcool, presente no ácido pipérico. Por apresentarem atividades contra insetos, o extrato dos frutos dessa espécie é largamente empregadas em pesquisas de controle de insetos pragas.

Com base nessas considerações, e tendo em vista os prejuízos do $C$. maculatus durante o armazenamento do feijão Vigna; os elevados preços dos defensivos químicos e seus problemas decorrentes do uso inadequado, com o presente trabalho, objetivou-se avaliar a eficácia de extratos hidroalcoólicos de $P$. nigrum no controle daquela espécie de caruncho pelo método do "vapor".

O trabalho foi realizado no Laboratório de Processamento e Armazenamento de Produtos Vegetais do Departamento de Engenharia Agrícola da Universidade Federal de Campina Grande, U.F.C.G., Campina Grande-PB.

O delineamento estatístico utilizado foi inteiramente casualizado com quatro repetições e esquema fatorial $3 \times 5$ (três extratos $\mathrm{x}$ cinco tempos de exposição aos extratos). Os dados foram submetidos às análises de variância e regressão.

Os extratos foram obtidos a partir de frutos de $P$. nigrum submetidos à secagem em estufa à temperatura constante de $40{ }^{\circ} \mathrm{C}$, durante $48 \mathrm{~h}$. Depois de secos, os frutos foram moídos em triturador de facas elétrico, pesado em balança analítica e guardado em recipiente escuro até a obtenção dos extratos.

O solvente utilizado na formulação dos extratos foi obtido a partir de diluição em álcool etílico a 92,8\% para $70 \%, 50 \%$ e $30 \%$, pela seguinte fórmula:

$$
\mathrm{X}=\frac{\mathrm{VG}^{\prime}}{\mathrm{G}}
$$

Em que:

$\mathrm{X}=$ Quantidade de solvente para o grau desejado;

$\mathrm{V}=$ Volume de álcool que se quer obter;

G'= Grau do álcool que se quer obter;

$\mathrm{G}=$ Grau do álcool a ser diluído.

Para formulação dos extratos, foram pesados $500 \mathrm{~g}$ da massa obtida na trituração, a qual foi, previamente, umedecida em um bequer com o solvente (álcool etílico a $70 \%, 50 \%$ e $30 \%$ ), para que o volume das células, que se encontrava reduzido, passasse a se expandir em contato com o solvente. A massa umedecida foi aos poucos transferida para o interior do percolador (extrator), o qual continha pequena quantidade de algodão hidrófilo ao fundo. Logo em seguida foi adicionado um disco de papel filtro e sobre este, um disco de metal perfurado, a fim de evitar que a adição do solvente levasse as partículas da massa já comprimida. Posteriormente, com o dispositivo (torneira) de saída do líquido extrator aberto, o solvente foi colocado dentro do percolador, pela parte superior, de maneira que ficasse de 2 a $3 \mathrm{~cm}$ de espessura até o início do gotejamento. Neste momento, a torneira foi fechada para que o material ficasse em repouso (maceração) por 24 horas e assim ocorrer a perfeita embebição da droga pelo solvente e a dissolução dos respectivos reconstituintes. Passado este período, o macerado foi percolado, em velocidade moderada de aproximadamente 20 gotas/minuto, até à obtenção do extrato bruto, o qual foi identificado e guardado em recipientes escuros.

A mortalidade dos insetos adultos foi verificada com base no princípio "tóxico" dos extratos aplicados na forma de vapor (ALMEIDA et al., 1999), durante 5, 10, 15, 20 e 25 minutos, e inalados pela traquéia e demais vias respiratórias. Foi utilizado um compressor adaptado com

Ciênc. agrotec., Lavras, v. 30, n. 4, p. 793-797, jul./ago., 2006 
capacidade de emitir partículas da ordem de 1 a 5 micra, onde o ar liberado pelo mesmo, ao passar por um recipiente levava o extrato nele contido, na forma de vapor diretamente para um recipiente plástico de $20 \mathrm{~cm}$ de altura por $12 \mathrm{~cm}$ de diâmetro, o qual continha 100 insetos adultos com três dias de idade. Estes recipientes tinham em suas tampas duas perfurações, uma para a entrada do "vapor" (extrato) e a outra para movimentação do oxigênio durante a aplicação.

A avaliação (número de insetos mortos) foi realizada 48 horas após a aplicação dos extratos, considerando-se vivos todos os insetos que moviam qualquer parte do corpo, mesmo aqueles que permanecem imóveis e só se moviam lentamente quando estimulados.

Os dados da análise de variância revelaram efeito significativo dos fatores extrato e tempo de exposição, bem como de sua interação. Desdobrando-se a interação extrato x tempo de exposição aos extratos, referente à mortalidade do caruncho adulto do feijão Vigna, as médias das porcentagens de mortalidade em função do tempo de exposição apresentaram melhor ajuste ao modelo quadrático de regressão, o que pode ser confirmado pelo maior valor de coeficiente de correlação, $\mathrm{R}^{2}$ (Tabela 1).

Mediante os dados expressos na Figura 1, observase que a mortalidade aumenta com o período de exposição do extrato (até os primeiros 15 minutos). A partir dos 20 minutos de exposição dos insetos aos extratos a mortalidade atingiu $100 \%$, independente da concentração do álcool utilizado. O comportamento desta variável foi descrito por um modelo quadrático de regressão com coeficiente de determinação $\left(\mathrm{R}^{2}\right)$ acima de $85 \%$, indicando bom ajuste aos dados (Figura 1). Boff \& Almeida (1995) observaram que a mortalidade de Sitotroga cerealella (Oliv., 1819)(Lepidóptera:Gelechiidae) causadas pelos extratos soxhlet acetônico e metanólico de $P$. nigrum foi superior a $90 \%$. Ainda segundo Boff \& Almeida (1996), extratos metanólicos e, ou acetônicos dessa piperácea, obtidos em aparelho de Soxhlet ou por maceração, mostraram efeito tóxico crescente sobre ovos de diferentes idades de $S$. cerealella.

TABELA 1 - Resumo das análises de variância e regressão para mortalidade de adultos de Callosobruchus maculatus, 48 horas após a exposição aos extratos de Piper nigrum.

\begin{tabular}{|c|c|c|c|}
\hline Fonte de variação & $\mathbf{G L}$ & QM & $\mathbf{R}^{2}$ \\
\hline $\begin{array}{l}\text { Extrato (E) } \\
\text { Tempo (T) } \\
\text { Ex T } \\
\text { Resíduo }\end{array}$ & $\begin{array}{c}2 \\
4 \\
8 \\
45\end{array}$ & $\begin{array}{c}41,3187^{* *} \\
87,9843^{* *} \\
9,4828^{* *} \\
1,01667\end{array}$ & \\
\hline $\mathrm{CV}(\%)$ & 1,03 & & \\
\hline \multicolumn{4}{|l|}{$30 \%$ (álcool etílico) } \\
\hline $\begin{array}{l}\text { Linear } \\
\text { Quadrática }\end{array}$ & $\begin{array}{l}1 \\
1\end{array}$ & $\begin{array}{l}260,1000^{* *} \\
31,5000^{* *}\end{array}$ & $\begin{array}{l}0,89 \\
0,99\end{array}$ \\
\hline $\mathrm{CV}(\%)$ & 0,89 & & \\
\hline \multicolumn{4}{|l|}{ 50\% (álcool etílico) } \\
\hline $\begin{array}{l}\text { Linear } \\
\text { Quadrática }\end{array}$ & $\begin{array}{l}1 \\
1\end{array}$ & $\begin{array}{l}62,5000^{* *} \\
25,7857^{* *}\end{array}$ & $\begin{array}{l}0,68 \\
0,97\end{array}$ \\
\hline $\mathrm{CV}(\%)$ & 1,05 & & \\
\hline \multicolumn{4}{|l|}{ 70\% (álcool etílico) } \\
\hline $\begin{array}{l}\text { Linear } \\
\text { Quadrática }\end{array}$ & $\begin{array}{l}1 \\
1\end{array}$ & $\begin{array}{l}22,5000^{* *} \\
16,0714^{* *}\end{array}$ & $\begin{array}{l}0,50 \\
0,85\end{array}$ \\
\hline
\end{tabular}

\footnotetext{
${ }^{* *}$ Significativo a $1 \%$ de probabilidade.
} 
Pode-se observar mediante os dados contidos na Tabela 2, que todos os extratos foram eficientes em matar o caruncho do feijão vigna causando mortalidade acima de $90 \%$ já no menor período de exposição $(5 \mathrm{~min})$. Em termos de valores absolutos (100\%), o extrato formulado com $70 \%$ de álcool etílico foi o mais agressivo, tendo matado todos os indivíduos de $C$. maculatus a partir de 10 minutos de exposição do extrato na forma de vapor. O extrato formulado com $50 \%$ de álcool etílico atingiu este controle quando o tempo de exposição foi igual ou superior a 15 minutos e o extrato com $30 \%$ de álcool etílico, a partir dos 20 minutos de exposição. De acordo com El-Nahal et al. (1989), o período de exposição dos extratos é mais importante que a dose aplicada. Rahman \& Schmidt (1999), em estudo do efeito de óleo de Acorus calamus na forma de vapor contra Callosobruchus phaseoli (Gyllenhal, 1833), constataram que a maior mortalidade desse inseto estava relacionada com o aumento do período de exposição do extrato.

Durante a aplicação dos extratos verificou-se que aos três minutos o comportamento dos insetos era de intensa agitação e que aos cinco minutos do início da aplicação, os mesmos não tinha, aparentemente, mais equilíbrio. Em estudo com Sitophilus spp., Almeida et al. (1999) verificaram esse mesmo comportamento em pouco tempo ( $\pm 10 \mathrm{~min}) \mathrm{de}$ exposição aos extratos, sendo que os insetos desprendiamse com facilidade da parte superior do recipiente. Estas observações estão relacionadas ao fato dos insetos possuírem respiração traqueal, a qual em número de 10 pares, abrem-se lateralmente através de pequenos orifícios

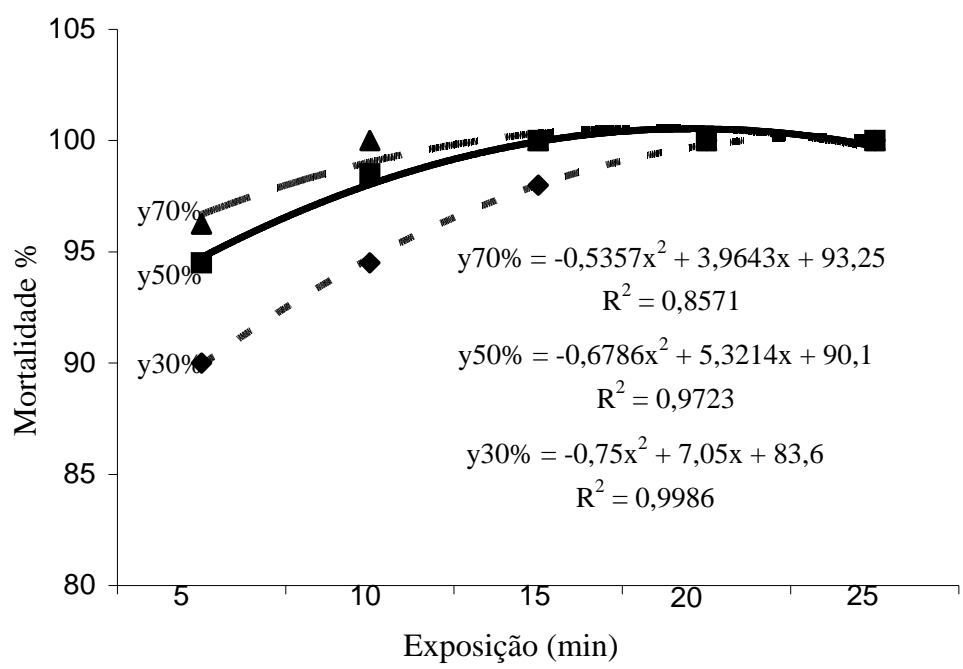

FIGURA 1 - Mortalidade de adultos de Callosobruchus maculatus com 3 dias de idade em função do tempo de exposição aos extratos de Piper nigrum formulados com 70, 50 e 30\% de álcool etílico, após 48 horas da exposição ao vapor.

TABELA 2 - Mortalidade (\%) de adultos de Callosobruchus maculatus após 48 horas da aplicação dos extratos Piper nigrum formulados com 70,50 e $30 \%$ de álcool etílico, na forma de vapor.

\begin{tabular}{|c|c|c|c|c|c|}
\hline \multirow[b]{2}{*}{ Tratamento } & \multicolumn{5}{|c|}{ Exposição do Extrato (min) } \\
\hline & 5 & 10 & 15 & 20 & 25 \\
\hline Ext. $30 \%$ & $90,00 \mathrm{c}$ & $94,50 \mathrm{~b}$ & $98,00 \mathrm{~b}$ & $100,00 \mathrm{a}$ & $100,00 \mathrm{a}$ \\
\hline Ext. $50 \%$ & $94,50 \mathrm{~b}$ & $98,50 \mathrm{a}$ & $100,00 \mathrm{a}$ & $100,00 \mathrm{a}$ & $100,00 \mathrm{a}$ \\
\hline Ext. $70 \%$ & 96,26 a & $100,00 \mathrm{a}$ & $100,00 \mathrm{a}$ & $100,00 \mathrm{a}$ & $100,00 \mathrm{a}$ \\
\hline
\end{tabular}

Dms para coluna $=1,7276$ (letras minúsculas).

*Médias seguidas pela mesma letra não diferem estaticamente pelo teste de Tukey, a nível de 1\% de probabilidade.

Ciênc. agrotec., Lavras, v. 30, n. 4, p. 793-797, jul./ago., 2006 
denominados estigmas. Em função desta estrutura física, os insetos absorvem e reagem rapidamente à aplicação dos extratos na forma de vapor, ocasionando, conseqüentemente, a morte dos mesmos por asfixia.

Conclui-se que a mortalidade dos insetos adultos aumenta com o incremento do período de exposição aos extratos. Todos os extratos de $P$. nigrum foram eficientes em matar o caruncho do feijão Vigna, embora, em termos absolutos, o extrato formulado com $70 \%$ de álcool etílico tenha sido o mais eficaz.

\section{REFERÊNCIAS BIBLIOGRÁFICAS}

ALMEIDA, F. A. C.; GOLDFARB, A. C.; GOUVEIA, J. P. G. de. Avaliação de extratos vegetais e métodos de aplicação no controle de Sitophilus spp. Revista Brasileira de Produtos Agroindustriais, Campina Grande, v. 1, n. 1, p. 13-20, 1999.

BOFF, M. I. C.; ALMEIDA, A. A. Efeito residual de Piper nigrum sobre larvas neonatas de Sitotroga cerealella (Lepidoptera: Gelechiidae). In: CONGRESSO BRASILEIRO DA SOCIEDADE ENTOMOLÓGICA DO BRASIL, 24., 1995. Anais... [S.1.]: SEB, 1995. p. 115-121.

BOFF, M. I. C.; ALMEIDA, A. A. Ação tóxica de pimenta do reino, Piper nigrum, em ovos de Sitotroga cerealella (Lepidoptera: Gelechiidae). In: CONGRESSO BRASILEIRO DA SOCIEDADE ENTOMOLÓGICA DO BRASIL, 25., 1996. Anais... [S.1.]: SEB, 1996. p. 423-429.

COSTA, A. F. Farmacognosia. Lisboa: Fundação Calovutegulben, 1994. v. 2.

CUNHA, E. M. Efeito de produtos vegetais e da fosfina no controle do Callosobruchus maculatus e na qualidade fisiológica de sementes de caupi (Vigna unguiculata). 2002. 37 f. Dissertação (Mestrado) - Universidade Federal da Paraíba, Areia, 2002.
DONGRE, T. K.; PAWAR, S. E.; THAKARE, R. G.; HARWALKAR, M. R. Identification of resistant sources to cowpea weevil (Callosobruchus maculatus) in Vigna spp. and inheritance of their resistance in black gram (Vigna var. mungo). Journal of Stored Sroducts Research, Elmsford, v. 32, p. 201-204, 1996.

EL-NAHAL, A. K. M.; SCHIMDT, G. H.; RISHA, E. M. Vapour of Acorus calamus oil a space treatment for storedproduct insects. Journal of Stored Products Research, Elmsford, v. 25, n. 4, p. 211-216, 1989.

FARONI, L. R. A.; MOLIN, L.; ANDRADE, E. T.; CARDOSO, E. G. Utilização de produtos naturais no controle de Acanthoscelides obtectus em feijão armazenado. Revista Brasileira de Armazenamento, Viçosa, v. 20, p. 44-48, 1995.

QUARLES, W. Botanical pesticides from Chenopodium. IPM Practitioner, [S.1.], v. 14, n. 2, p. 1-11, 1992.

RAHMAN, M. M.; SCHMIDT, G. H. Effect of Acorus calamus (Araceae) essential oil vapours from various origins on Callosobruchus phaseoli (Coleoptera: Bruchidae). Journal of Stored Products Research, Elmsford, v. 35, p. 285-295, 1999.

SECK, D.; SIDIBÉ, R.; HAUBRUGE, E.; HEMPTINNE, I.; GASPAR, C. La protection chimique des stocks de niébé et de maís contre les insectes au Sénégal. Medelingen van de Faculteit Landbouwwetenschappen Rijksuniversiteit Gen, [S.1.], p. 1225-1234, 1991.

TANZUBIL, P. B. Control of some insect pests of cowpea (Vigna unguiculata) with neem (Azadirachta indica) in Northern Ghana. Tropical Pest Management, Basingstoke, v. 37, p. 216-217, 1991. 